\title{
Abdominal rebleeding after transcatheter arterial embolization for ruptured pseudoaneurysms associated with severe acute pancreatitis: a retrospective study
}

\author{
Min Ai, DaZhi Gao, GuangMing Lu, Jian Xu \\ Department of Medical Imaging, Jinling Hospital, Clinical School of Medical College, Nanjing University, Nanjing, Jiangsu Province, China
}

Videosurgery Miniinv 2021; 16 (1): 83-90

DOI: https://doi.org/10.5114/wiitm.2020.97426

\begin{abstract}
Introduction: Patients are at risk of abdominal rebleeding after transcatheter arterial embolization (TAE) for pancreatitis-related pseudoaneurysm, which increases the mortality rate.

Aim: This study was performed to evaluate the effects of an intestinal fistula (IF) and the anatomical location of the pseudoaneurysm on abdominal rebleeding after TAE of a ruptured pseudoaneurysm associated with severe acute pancreatitis (SAP).

Material and methods: From February 2013 to November 2019, 24 patients with SAP-related pseudoaneurysm rupture and hemorrhage in our hospital underwent TAE. All patients' epidemiological data and related medical histories were collected and statistically analyzed. We classified the pseudoaneurysms as type I, II, and III according to their anatomical locations and as type A (without an IF) and type B (with an IF).

Results: The interventions for abdominal infection in patients with type I pseudoaneurysms were percutaneous drainage in 6 patients, endoscopic necrotic tissue removal in 5, and surgical necrotic tissue removal or enterostomy in none, with a rebleeding rate of 33.3\% (3/9 patients). The interventions for abdominal infection in patients with type II pseudoaneurysms were percutaneous drainage in 7 patients, endoscopy in three, and surgery in one, with a rebleeding rate of $20.0 \%$ (2/10 patients). The interventions for abdominal infection in patients with type III pseudoaneurysms were percutaneous drainage in 3 patients, endoscopy in 1, and surgery in 2, with a rebleeding rate of $80.0 \%$ (4/5 patients). There was no statistically significant difference in the types of interventions for abdominal infection among patients with type I, II, and III pseudoaneurysms $(p=0.355)$ or in the rate of abdominal rebleeding after TAE for type III pseudoaneurysms $(p=0.111)$. The interventions for abdominal infection in patients with type $A$ pseudoaneurysms were percutaneous drainage in 13 patients, endoscopy in 6, and surgery in 1, with a rebleeding rate of $22.2 \%$ (4/18 patients) and mortality rate of $11.1 \%$ (2/18 patients). The interventions for abdominal infection in patients with type B pseudoaneurysms were percutaneous drainage in 3 patients, endoscopy in 3 , and surgery in 2 , with a rebleeding rate of $83.3 \%$ (5/6 patients) and mortality rate of $66.7 \%$ (4/6 patients). There was no significant difference in the types of interventions for abdominal infection in patients with and without IF ( $p=0.215)$; however, the rebleeding rate and mortality rate were significantly higher in patients with IF ( $p=0.015$ and 0.018 , respectively). Conclusions: IF may increase the rate of abdominal rebleeding after TAE for ruptured SAP-related pseudoaneurysms, while the anatomical location of the pseudoaneurysm may not affect the rate of rebleeding after TAE.
\end{abstract}

Key words: rebleeding, intestinal fistula, anatomical position, pseudoaneurysm, severe acute pancreatitis.

\footnotetext{
Address for correspondence

Jian Xu, Department of Medical Imaging, Jinling Hospital, Clinical School of Medical College, Nanjing University, Nanjing 210002,

Jiangsu Province, China, phone: 13851656307, fax: 025-84824051, e-mail: 13851656307@163.com
} 


\section{Introduction}

Pancreatitis is complicated by pseudoaneurysms in about $10 \%$ of cases [1]. This mainly occurs by continuous erosion of the adjacent arterial wall by trypsin and inflammatory fluid in the abdominal cavity; the vessel wall becomes thinner and gradually expands under the impact of blood flow to form a pseudoaneurysm [2]. Pseudoaneurysm rupture in patients with pancreatitis is rare, but if untreated, the mortality rate can be as high as $40 \%$ to $100 \%$ [2-4]. Transcatheter arterial embolization (TAE) is a first-line treatment option for pancreatitis-associated pseudoaneurysm rupture because of its high success rate and low mortality rate [5-8]. However, patients who have undergone TAE are at risk of abdominal rebleeding, which increases the mortality rate $[9,10]$.

Pang et al. [10] described three types of pseudoaneurysms according to their anatomical location, presence or absence of intestinal communication, and presence or absence of contact with pancreatic fluid. This classification has greatly helped clinicians identify patients with a high risk of rebleeding after embolization for pancreatitis-associated pseudoaneurysm rupture. In this way, clinicians can make appropriate decisions to reduce the risk of rebleeding after embolization and reduce patient mortality. However, no specific clinical cases were described by Pang. In the present study, we referred to the classification proposed by Pang and retrospectively collected the relevant treatment data of 24 patients with severe acute pancreatitis (SAP) complicated by pseudoaneurysm rupture and bleeding in our hospital for the performance of a statistical analysis.

\section{Aim}

This study was performed to evaluate the effects of an intestinal fistula (IF) and the anatomical location of the pseudoaneurysm on abdominal rebleeding after transcatheter arterial embolization (TAE) of a ruptured pseudoaneurysm associated with severe acute pancreatitis (SAP).

\section{Material and methods}

From February 2013 to November 2019, 24 patients with SAP were treated in our hospital, all of whom underwent TAE for a ruptured pseudoaneurysm. Epidemiological data, TAE embolism data, and relevant clinical data of all patients were collected by consulting the electronic medical records. Because this was a retrospective study, ethics approval from our institution was not required. Approval was obtained from the local ethics committee and all patients provided written informed consent before undergoing TAE.

After puncture of the femoral artery with the Seldinger technique, a 5-Fr sheath (Terumo Corporation, Tokyo, Japan) was inserted in the femoral artery. A 5-Fr angiographic catheter (RH catheter; Cook Medical, Bloomington, IN, USA) was then inserted through the sheath and successively inserted into the celiac artery, superior mesenteric artery, and inferior mesenteric artery. Digital subtraction angiography was performed and divided into the arterial phase, parenchymal phase, and venous phase. A pseudoaneurysm was suspected when the artery showed nodular dilatation upon filling with contrast agent. In such cases, a 2.6-Fr Stride microcatheter (Asahi Intecc Co., Ltd., Aichi, Japan) was immediately inserted into the vessel.

When the primary artery of the pseudoaneurysm was a large vessel of the celiac branch (gastroduodenal artery or splenic artery), a coil (Cook Medical) was used to successively fill the distal and proximal ruptures of the pseudoaneurysm using the 2.6-Fr Stride microcatheter. Finally, an appropriate amount of absorbable gelatin particles (Jiangxi Xiangen Medical Technology Development Co., Ltd., Jiangxi, China) was administered for proximal embolization to slow the blood flow and accelerate the formation of a thromboembolus in the spring coil. If the offending artery was a small branch vessel and the microcatheter could not be inserted selectively, embolization was performed with an appropriate amount of absorbable gelatin particles. The final angiogram revealed no development of a pseudoaneurysm in the lumen, and the embolization was considered complete.

The pseudoaneurysms were classified into three types according to their anatomical location [10]: type I arises from a minor artery but must be $5 \mathrm{~mm}$ away from its junction of origin from a type II or III artery; type II arises from a major artery that may be sacrificed without physiological consequences (e.g., splenic or gastroduodenal artery); and type III arises from a major artery that causes significant complications when sacrificed (e.g., superior mesenteric artery or hepatic artery proper). The pseudoaneurysms were classified into two types according to the pres- 
ence or absence of a gastrointestinal fistula [10]: type $A$ is not associated with a gastrointestinal fistula and type B is associated with a gastrointestinal fistula. Finally, we classified the pseudoaneurysms according to their anatomical location (type I, II, and III) and the presence or absence of an intestinal fistula (IF) (no patients in this study had a gastric fistula).

Three interventions for abdominal infection were used in this study: percutaneous peritoneal drainage, endoscopic clearance of necrotic tissue, and surgical removal of necrotic tissue or enterostomy under general anesthesia.

IFs are defined as pathological connections between any portion of the intestine and necrotic cavities, peritoneal cavities, retroperitoneal areas, or other internal organs [11]. With reference to a previous report [12], we injected contrast agent through the peritoneal drainage tube and diagnosed an IF if intestinal development was found.

Table I. Demographics and medical histories of 24 patients who underwent TAE for management of SAP-related bleeding

\begin{tabular}{|lc|}
\hline Parameter & Results \\
\hline Number of patients & 24 \\
\hline Age [years], mean (range) & $48(20-70)$ \\
\hline Male : female ratio & $18: 6$ \\
\hline Causes of SAP: & $58.3 \%(14 / 24)$ \\
\hline Biliary & $25 \%(6 / 24)$ \\
\hline Hyperlipidemic & $4.2 \%(1 / 24)$ \\
\hline Alcoholic & $4.2 \%(1 / 24)$ \\
\hline Postsurgical (total gastrectomy) & $8.3 \%(2 / 24)$ \\
\hline Unknown & $70.8 \%(17 / 24)$ \\
\hline Clinical presents before TAE: & $4.2 \%(1 / 24)$ \\
\hline \begin{tabular}{l} 
Bleeding from drains \\
\hline Melena
\end{tabular} & $4.2 \%(1 / 24)$ \\
\hline \begin{tabular}{l} 
Hematemesis \\
\hline Asymptomatic
\end{tabular} & $20.8 \%(5 / 24)$ \\
\hline \begin{tabular}{l} 
Laboratory abnormalities [U/l]: \\
\hline Mean amylase before TAE
\end{tabular} & $315(24-1423)$ \\
\hline Mean lipase before TAE & $38-105)$ \\
\hline Mean Hospital stay [days] & \\
\hline severe acute pancreatitis, TAE- transcatheter arterial embolization.
\end{tabular}

Embolization of the pseudoaneurysm was considered successful according to three criteria: the last intraoperative angiogram during TAE showed no contrast agent filling in the pseudoaneurysm cavity; signs of abdominal bleeding (bleeding from the abdominal drainage tube, hematemesis, and black stool) ceased after TAE, and the patient's vital signs improved; and postoperative follow-up of TAE with enhanced abdominal computed tomography showed no displacement of the spring coil and no contrast agent filling in the cavity of the pseudoaneurysm.

\section{Statistical analysis}

SPSS version 22 (IBM Corp., Armonk, NY, USA) was used for the statistical analysis. The intervention measures for abdominal infection in the different groups of patients with the two subtypes of pseudoaneurysm were compared by Fisher's exact probability test for a bidirectional unordered row list. The incidence and mortality rate of abdominal rebleeding in the different groups of patients with the two subtypes of pseudoaneurysm were compared by Fisher's exact probability test. A $p$-value of $\leq 0.05$ was considered statistically significant.

\section{Results}

The patients' epidemiologic characteristics, clinical manifestations, and laboratory indicators are

Table II. Patients with abdominal rebleeding

\begin{tabular}{|lcc|}
\hline Patient no. & Initial DSA & Second DSA \\
\hline 1 & GDA & SplA \\
\hline 2 & SMA & SMA $^{*}$ \\
\hline 3 & SplA & SMA \\
\hline 4 & $\begin{array}{c}\text { Right gastroepiploic } \\
\text { artery }\end{array}$ & SplA \\
\hline 5 & CHA & SplA \\
\hline 6 & SMA & - \\
\hline 7 & SplA & - \\
\hline 8 & SplA & - \\
\hline 9 & RHA & - \\
\hline
\end{tabular}

${ }^{*}$ Different anatomical positions. GDA - gastroduodenal artery, SplA - splenic artery, SMA - superior mesenteric artery, CHA - common hepatic artery, RHA - right hepatic artery, DSA - digital subtraction angiography. 
shown in Table I. Most of the patients with SAP were male (18/24 patients). Biliary pancreatitis was the main cause $(58.3 \% ; 14 / 24)$, and bleeding from the abdominal drainage tube was the most common symptom of pseudoaneurysm rupture $(70.8 \%$; 17/24).

The data of the 9 patients with SAP who developed peritoneal rebleeding are shown in Table II. In 5 of these patients, the location of the pseudoaneurysm differed from that on the primary angiogram. The other 4 patients did not undergo TAE again because their vital signs were extremely unstable. These patients eventually died of hypovolemic shock
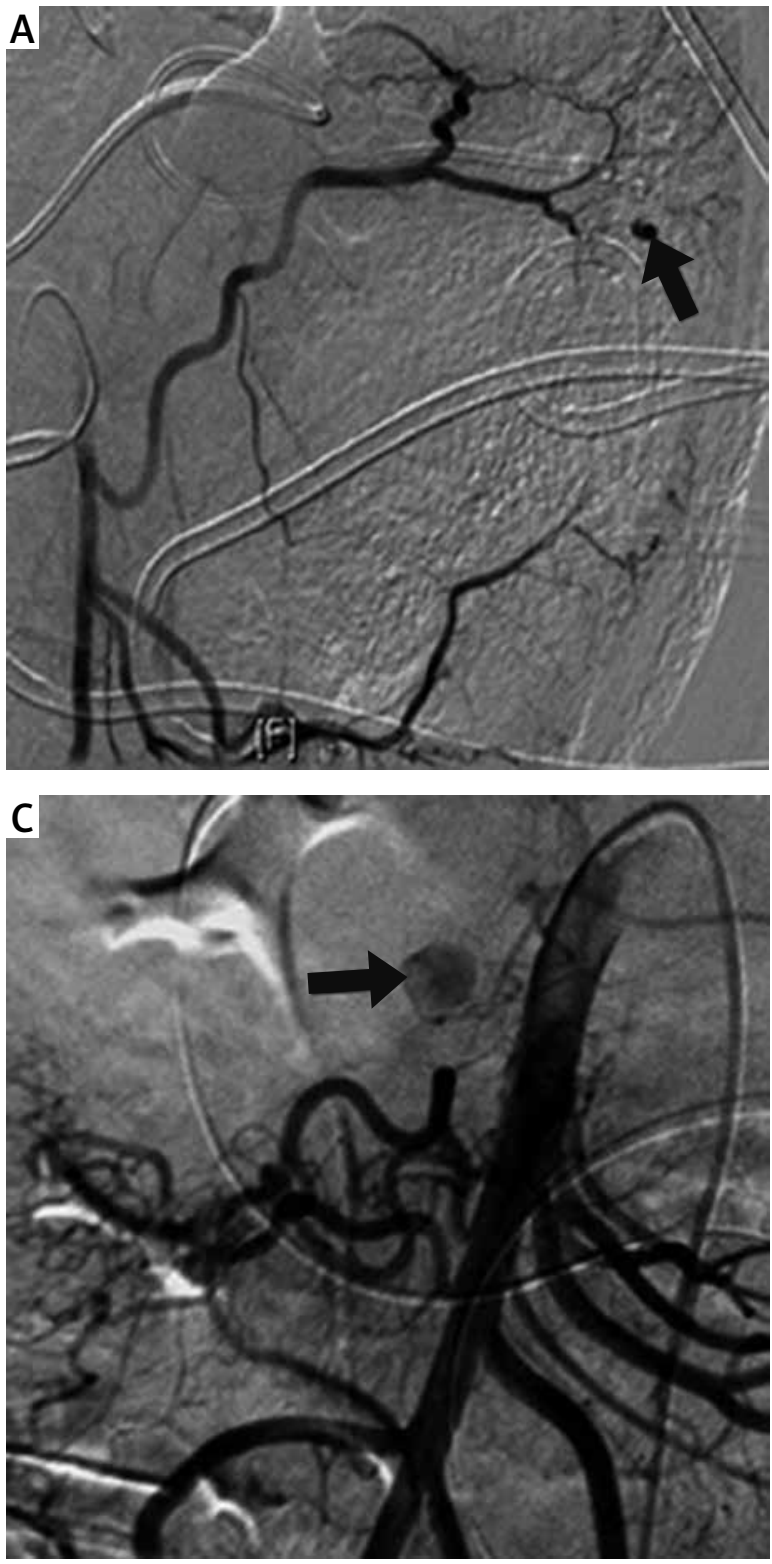

$(n=2)$, septic shock $(n=1)$, and multiple organ failure $(n=1)$.

The interventions for intraperitoneal infection and the rates of postoperative abdominal rebleeding in patients with type I (Photo $1 \mathrm{~A}$ ), II (Photo $1 \mathrm{~B}$ ), and III (Photo $1 \mathrm{C}$ ) pseudoaneurysms are shown in Table III. Compared with the other interventions for intraperitoneal infection, peritoneal drainage was performed in a higher proportion of cases in all three groups of patients $(54.5 \%, 63.6 \%$, and $50.0 \%$, respectively). The F-test revealed no statistically significant difference in the types of interventions for abdominal infection among patients with type I, II,

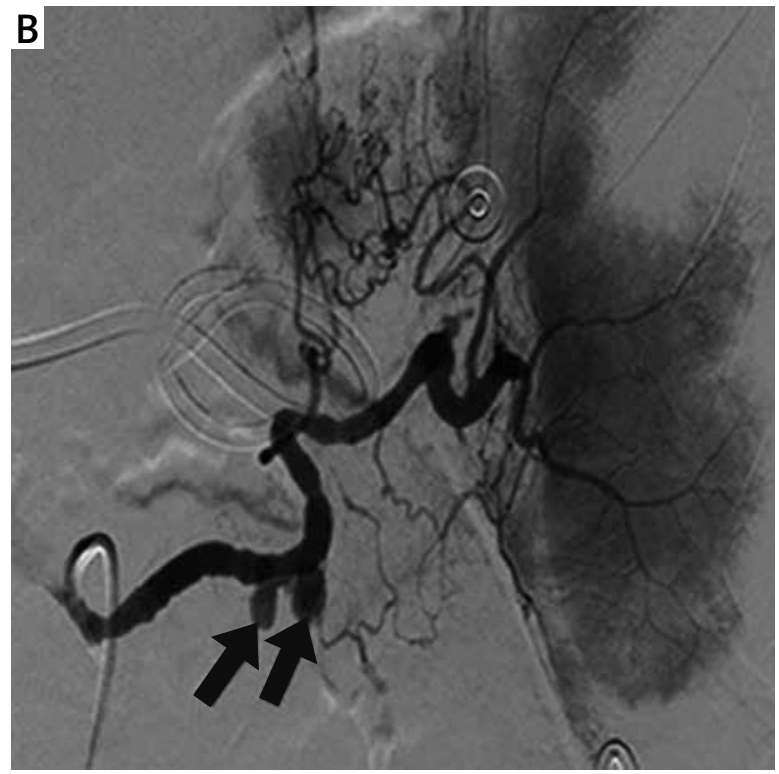

Photo 1. Three types of pseudoaneurysm according to their anatomical location. A - A 38year-old man with severe acute pancreatitis (SAP) with inferior mesenteric artery pseudoaneurysm formation (type I) (black arrow). B - A 36-year-old man with SAP with splenic artery pseudoaneurysm formation (type II) (black arrow). C - A 34-year-old man SAP with superior mesenteric artery pseudoaneurysm formation (type III) (black arrow) 
Table III. Interventions for abdominal infection and rates of abdominal rebleeding in patients with type I, II, and III SAP-associated pseudoaneurysms after TAE

\begin{tabular}{|c|c|c|c|c|c|c|c|}
\hline \multirow{2}{*}{$\begin{array}{l}\text { Anatomical } \\
\text { position }\end{array}$} & \multicolumn{3}{|c|}{ Intervention } & \multirow[t]{2}{*}{ Total } & \multicolumn{2}{|c|}{ Rebleeding } & \multirow[t]{2}{*}{ Total } \\
\hline & $\mathrm{a}$ & $b$ & c & & + & - & \\
\hline । & 6 & 5 & 0 & 11 & $3(33.3 \%)$ & 6 & 9 \\
\hline ॥ & 7 & 3 & 1 & 11 & $2(20.0 \%)$ & 8 & 10 \\
\hline III & 3 & 1 & 2 & 6 & $4(80.0 \%)$ & 1 & 5 \\
\hline Total & 16 & 9 & 3 & 28 & 9 & 15 & 24 \\
\hline
\end{tabular}

$a$ - peritoneal drainage, $b$ - endoscopy, $c$ - surgery (necrosectomy, terminal ileostomy). SAP-severe acute pancreatitis, TAE - transcatheter arterial embolization.

and III SAP-associated pseudoaneurysms $(p=0.355)$ and no significant difference in the rate of abdominal rebleeding after TAE for type III SAP-associated pseudoaneurysms ( $p=0.111 ; 33.3 \%$ vs. $20.0 \%$ vs. 80.0\%).

The interventions for intraperitoneal infection, the postoperative abdominal rebleeding rate, and the mortality rate of patients with SAP-associated type $A$ and $B$ (Photo 2) pseudoaneurysms are shown in Table IV. The F-test revealed no significant difference in the types of interventions for abdominal infection between patients with SAP-associated type $A$ and $B$ pseudoaneurysms ( $p=0.215)$; however, the bleeding recurrence rate and mortality rate were significantly higher in type B than A $(p=0.015$ and 0.018 , respectively) (22.2\% vs. $83.3 \% ; 11.1 \%$ vs. $66.7 \%)$.

\section{Discussion}

In this study, we referred to the classification method for pseudoaneurysms described by Pang et al. [10]. Among the three classification parameters described in their system, we selected two for use in this study: the anatomical location and presence/absence of an IF. These were chosen for the following reasons. First, type $A$ and $B$ pseudoaneurysms as defined by Pang refer to direct communication between a pseudoaneurysm at a fixed location and a gastrointestinal fistula, and the pseudoaneurysm is at risk of rerupture; however, this may have limitations in patients with SAP. In the present study, the second angiogram of 5 patients with abdominal rebleeding showed that the anatomical location of the pseudoaneurysm differed from that on the first angiogram. This suggests that after the first TAE for a ruptured pseudoaneurysm, the patient is at risk of bleeding caused by rupture of other intra-abdominal arteries or pseudoaneurysms that do not directly communicate with the IF. Therefore, we directly analyzed the effect of the presence or absence of an IF (i.e., whether or not the pseudoaneurysm directly communicates with the IF) on abdominal rebleeding after TAE of SAP-associated pseudoaneurysm rupture. Second, some patients with SAP in this study did not undergo an enhanced computed tomography examination before TAE; therefore, we were unable to determine whether the pseudoaneurysm was in direct contact with a pancreatic secretion-rich pseudocyst. As a result, we did not include factors associated with pancreatic fluid contact.

Rana et al. [13] found that most patients who had acute pancreatitis with abdominal bleeding had a history of an intervention for abdominal infection (such as percutaneous puncture drainage, endoscopic drainage, or surgical procedures). Szeliga and Jackowski [14] found that a surgical operation to remove an abdominal infection is associated with more severe complications (such as hemorrhage and wound infection) than minimally invasive drainage. Therefore, considering that the type of intervention for abdominal infection may affect the incidence of rebleeding after TAE in patients with SAP, we statistically compared the interventions for abdominal infection in different groups of patients with the two subtypes of pseudoaneurysms and found no significant difference between the two subtypes. We then analyzed whether the IF and the anatomical position of the pseudoaneurysm affected abdominal rebleeding after embolization of the SAP-associated pseudoaneurysm.

An IF may be an important cause of rebleeding after embolization of SAP-related pseudoaneurysms. Abdominal bleeding in patients with SAP can induce 

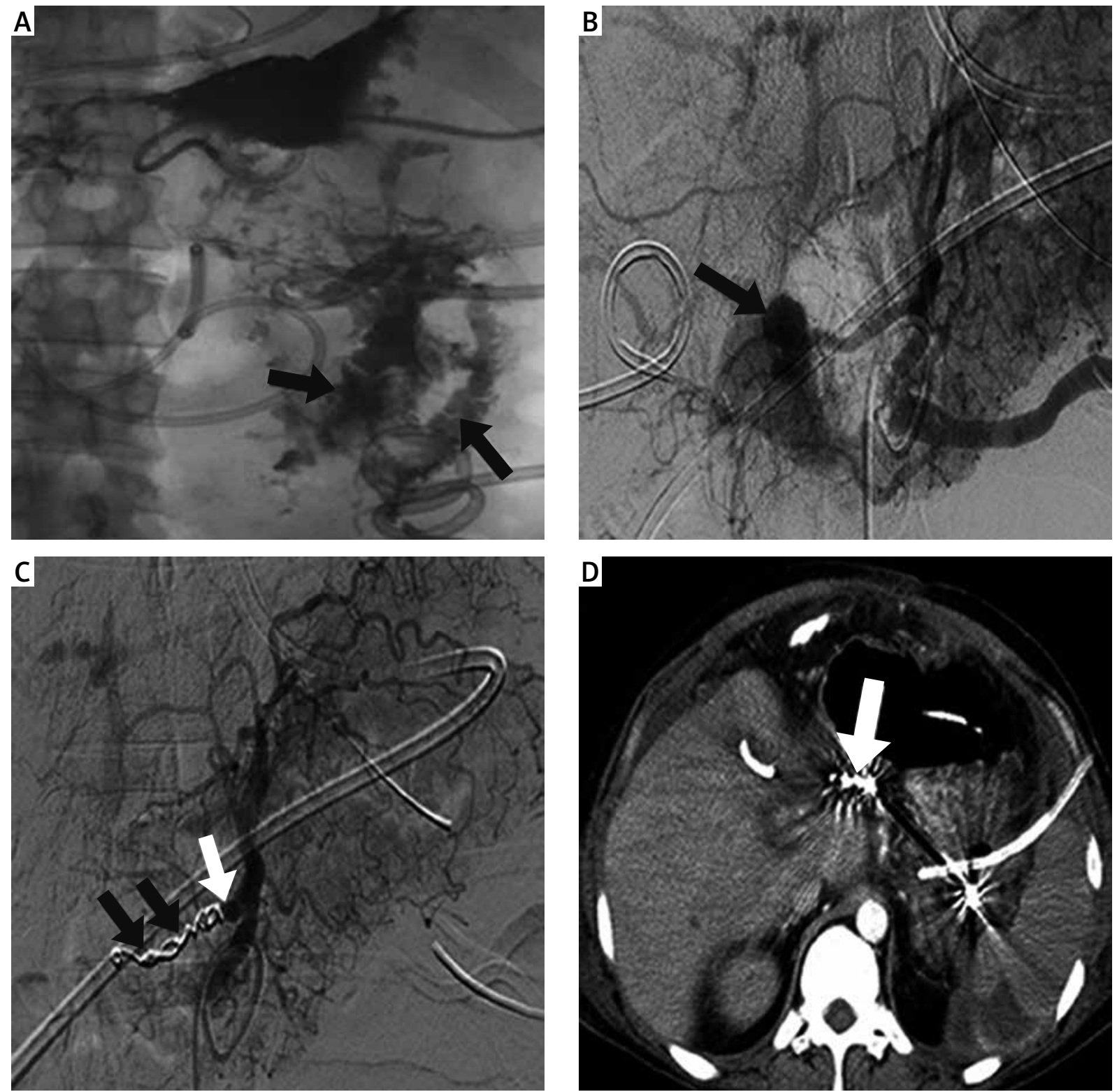

Photo 2. Embolization of a common hepatic artery pseudoaneurysm in a 30-year-old man with severe acute pancreatitis who developed bleeding from the abdominal drainage tube. A - In the fluoroscopic examination, contrast was seen in the left lower abdominal bowel (black arrow) after injection of contrast agent through the upper abdominal drainage tube. B - Angiographic images from the celiac trunk showed a cystic bulge of the common hepatic artery upon filling of contrast agent. A pseudoaneurysm (black arrow) was suspected. C - Celiac trunk reexamination showed embolization of the distal and proximal spring coil (black arrow) of the pseudoaneurysm, development of the stump of the common hepatic artery (white arrow), and no development of the pseudoaneurysm. D - After 1 month, an enhanced computed tomography scan of the upper abdomen showed that the coil (white arrow) was in place with no displacement and that no contrast agent was present in the cavity of the pseudoaneurysm

an IF by leading to ischemic necrosis of the intestinal wall; however, it can also aggravate abdominal infection, which has been found to be a high-risk trigger of IF [11]. Several studies have been performed to determine whether an IF affects abdominal bleeding. One study showed that about $60 \%$ of patients 
Table IV. Interventions for abdominal infection and rates of abdominal rebleeding and mortality after TAE in patients with type A and B SAP-associated pseudoaneurysms

\begin{tabular}{|c|c|c|c|c|c|c|c|c|c|c|}
\hline \multirow[t]{2}{*}{ IF } & \multicolumn{3}{|c|}{ Intervention } & Total & \multicolumn{2}{|c|}{ Rebleeding } & Total & \multicolumn{2}{|c|}{ Death } & Total \\
\hline & $\mathrm{a}$ & $b$ & c & & + & - & & + & - & \\
\hline A & 13 & 6 & 1 & 20 & $4(22.2 \%)$ & 14 & 18 & 2 (11.1\%) & 16 & 18 \\
\hline B & 3 & 3 & 2 & 8 & 5 (83.3\%) & 1 & 6 & $4(66.7 \%)$ & 2 & 6 \\
\hline Total & 16 & 9 & 3 & 28 & 9 & 15 & 24 & 6 & 18 & 24 \\
\hline
\end{tabular}

$A$ - patients without intestinal fistula; $B$ - patients with intestinal fistula; $a$ - peritoneal drainage, $b$ - endoscopy, $c$ - surgery (necrosectomy, terminal ileosto$m y), S A P$ - severe acute pancreatitis, TAE - transcatheter arterial embolization, IF - intestinal fistula.

with a pancreaticointestinal fistula had gastrointestinal bleeding [15]. In addition, a literature review by Pang et al. [10] showed that an IF can increase the risk of rebleeding after abdominal hemorrhage embolization. In the present study, the rate of bleeding recurrence after embolization was significantly higher in patients with than without an IF (83.3\% vs. $22.2 \%$, respectively). We speculate that the IF aggravated the abdominal infection, leading to increased accumulation of infectious and corrosive fluid in the abdominal cavity; this fluid directly invaded the pseudoaneurysm wall in contact with it, resulting in rupture and bleeding.

An IF may increase mortality in patients with SAP. However, some studies of patients with SAP have shown no statistically significant difference in mortality between those with and without an IF. This lack of significance is related to the following three points. First, the sinus of the IF is mostly confined to the retroperitoneal space, and diffusion is limited. Second, when the abdominal infection is controlled, the sinus tract usually heals on its own. Third, improvements in surgical techniques and parenteral nutrition have also improved the healing of IFs [11, 16]. In the present study, the mortality rate was significantly higher in patients with than without IFs (66.7\% vs. $11.1 \%$, respectively), which differs from the findings of previous reports. We consider that this is related to the fact that all IFs in this study were diffuse. A diffuse IF is associated with more serious complications and a poorer prognosis than a localized IF, which may lead to increased mortality $[17,18]$.

The anatomic location of the pseudoaneurysm may not affect the rebleeding rate after TAE in patients with SAP. Hur et al. [19] found that patients who undergo pancreaticoduodenectomy are prone to develop a pancreatic fistula, leading to dissemi- nated trypsin infiltration into the abdominal cavity; the trypsin then corrodes the vascular walls in the abdominal cavity. Thus, in addition to the gastroduodenal arteries (the arteries directly affected by the pancreaticoduodenectomy procedure itself), any external hepatic artery in contact with extravasated trypsin is at risk of rupture by corrosion. Abdominal inflammation and necrotizing fluid accumulation can reportedly increase the risk of abdominal bleeding in patients with acute pancreatitis, and SAP is even more likely to cause such complications [20]. We found no difference in the rate of abdominal rebleeding after embolization of the SAP-associated pseudoaneurysms among the three types of anatomical locations in the present study. This finding suggests that after rupture and embolization of the pseudoaneurysm in a small branch artery, the patients were still at risk of abdominal rebleeding; this may have been related to the presence of SAP in all patients of this study.

This study had several limitations. First, it was a retrospective study, which limits the credibility of the conclusions. Second, because of the small number of patients, the optimal intervention time and sequence of measures for treatment of the abdominal infection source could not be determined. Third, although an IF may increase the risk of rebleeding and death in patients with SAP, it is self-limiting; thus, whether intervention is necessary and if so, whether endoscopic or surgical intervention is the optimal treatment choice cannot be determined. These questions require further study.

\section{Conclusions}

Pseudoaneurysm rupture is a rare but fatal complication of SAP. Abdominal rebleeding after TAE for pseudoaneurysms increases the risk of death. An IF may increase the risk of abdominal rebleeding after 
embolization of a ruptured pseudoaneurysm in patients with SAP. The anatomic location of an SAP-related pseudoaneurysm may not affect peritoneal rebleeding.

\section{Conflict of interest}

The authors declare no conflict of interest.

\section{References}

1. Balhazar EJ, Fisher LA. Hemorrhagic complications of pancreatitis: radiologic evaluation with emphasis on $\mathrm{CT}$ imaging. Pancreatology 2001; 1: 306-13.

2. Verde F, Fishman EK, Johnson PT. Arterial pseudoaneurysms complicating pancreatitis: literature review. J Comp Assist Tomogr 2014; 39: 7-12.

3. Saad NE, Saad WE, Davies MG, et al. Pseudoaneurysms and the role of minimally invasive techniques in their management. RadioGraphics 2005; 25: 173-89.

4. Seetharama MK, Ananthashayana VH, Shivanand G, et al. Interventional radiology in the management of visceral artery pseudoaneurysms: a review of techniques and embolic materials. Korean J Radiol 2016; 17: 351-63.

5. Mijnsbrugge WV, Laleman W, Steenbergen WV, et al. Long-term clinical and radiological outcome of endovascular embolization of pancreatitis-related pseudoaneurysms. Acta Radiologica 2016; 58: 316-22.

6. Ai M, Lu GM, Xu J. Endovascular embolization of arterial bleed ing in patients with severe acute pancreatitis. Videosurgery Miniinv 2019; 14: 401-7.

7. NykäNen T, Udd M, Peltola EK, et al. Bleeding pancreatic pseudoaneurysms: management by angioembolization combined with therapeutic endoscopy. Surg Endosc 2017; 31: 692-703.

8. Czernik M, Stefańczyk L, Szubert W, et al. Endovascular treatment of pseudoaneurysms in pancreatitis. Videosurgery Miniinv 2014; 13: 138-44.

9. Carr JA, Cho JS, Shepard AD, et al. Visceral pseudoaneurysms due to pancreatic pseudocysts: rare but lethal complications of pancreatitis. J Vasc Surg 2000; 32: 722-30.

10. Pang TCY, Maher R, Gananadha S, et al. Peripancreatic pseudoaneurysms: a management-based classification system. Surg Endosc 2014; 28: 2027-38.

11. Hua Z, Su Y, Huang X, et al. Analysis of risk factors related to gastrointestinal fistula in patients with severe acute pancreatitis: a retrospective study of 344 cases in a single Chinese center. BMC Gastroenterology 2017; 17: 29.

12. Falconi M, Pederzoli P. The relevance of gastrointestinal fistulae in clinical practice: a review. Gut 2001; 49: iv2-10.

13. Rana SS, Sharma V, Bhasin DK, et al. Gastrointestinal bleeding in acute pancreatitis: etiology, clinical features, risk factors and outcome. Trop Gastroenterol 2015; 36: 31-5.

14. Szeliga J, Jackowski M. Minimally invasive procedures in severe acute pancreatitis treatment-assessment of benefits and possibilities of use. Videosurgery Miniinv 2014; 9: 170-8.

15. Berne TV, Edmondson HA. Colonic fistulization due to pancreatitis. Am J Surg 1966; 111: 359-63.
16. Shen D, Ning C, Huang G, et al. Outcomes of infected pancreatic necrosis complicated with duodenal fistula in the era of minimally invasive techniques. Scand I Gastroenterol 2019; 54: 766-72.

17. Kochhar R, Jain K, Gupta V, et al. Fistulization in the GI tract in acute pancreatitis. Gastrointest Endosc 2012; 75: 436-40.

18. Gardner A, Gardner G, Feller E. Severe colonic complications of pancreatic disease. J Clin Gastroenterol 2003; 37: 258-62.

19. Hur S, Yoon CJ, Kang SG, et al. Transcatheter arterial embolization of gastroduodenal artery stump pseudoaneurysms after pancreaticoduodenectomy: safety and efficacy of two embolization techniques. J Vasc Interv Radiol 2011; 22: 294-301.

20. Chen Y, Zhou J, Li G, et al. Early spontaneous abdominal bleeding is associated with poor outcome in moderate to severe acute pancreatitis patients: a propensity matched study. Sci Rep 2017; 7: 42607.

Received: 26.03.2020, accepted: 19.05.2020. 